\title{
SINGULAR DEGENERATIONS OF CALABI-YAU MANIFOLDS AND THE WEIL-PETERSSON METRIC
}

\author{
PAUL S. GREEN
}

(Communicated by Jonathan M. Rosenberg)

\begin{abstract}
Degenerations of Calabi-Yau manifolds with isolated singularities somewhat more general than nodes are shown to be at finite distance from nonsingular manifolds in the Weil-Petersson metric on the appropriate moduli spaces.
\end{abstract}

If $\mathscr{M}(z)$ is a nonsingular complex threefold with trivial canonical bundle and holomorphic three-form $\Omega_{z}$, depending holomorphically on $z \in \mathscr{Z}$, for some complex manifold $\mathscr{Z}$, then $\log \left(\int_{\mathscr{M}(z)} \Omega_{z} \wedge \bar{\Omega}_{z}\right)$ is the Kähler potential for a pseudo-metric on $\mathscr{Z}$, which is a metric provided $\mathscr{Z}$ is an effective deformation space in the sense of [2]. This metric has come to be known in the physics literature as the Weil-Petersson metric, although the strict analogy with moduli spaces of curves holds only for curves of genus one.

This paper is a step in attempting to understand the completion of this WeilPetersson metric by studying its behaviour with respect to singular degenerations. In particular, we study the case where $\mathscr{Z}$ is a punctured disk, and the holomorphic family $\{\mathscr{M}(z)\}$ can be completed by the inclusion of a variety $\mathscr{M}(0)$ with isolated singularities. We give some sufficient conditions for curves through the deleted point to have finite length. The case of nodal degenerations was discussed in [1].

We proceed mainly by studying the periods of $\Omega_{z}$. We shali denote by $C(z)$ a locally constant homology class in $H_{3}(\mathscr{M}(z)){ }^{1}$

$$
\int_{C(z)} \Omega_{z}
$$

is a holomorphic, although possibly multiple valued, function of $z$. We shall usually assume that $z$ ranges over a punctured disk, $D-\{0\}$, and write $\mu$ for the monodromy operator on $H_{3}(\mathscr{M}(z))$. Our principal results, Theorems

Received by the editors April 5, 1989 and, in revised forms, April 25, 1989 and March 16, 1990. 1980 Mathematics Subject Classification (1985 Revision). Primary 14J30, 32G13, 53C25.

Key words and phrases. Calabi-Yau, Weil-Peterssen, singular degeneration.

${ }^{1}$ We think of the parameter $z$ somewhat ambiguously as an element either of $\mathscr{Z}$ or of its universal covering space. 
A and B below, generalize the case of a nodal degeneration, for which $\mu$ is a transvection and $(1-\mu)^{2}=0$.

Theorem A. Let $\left\{\left(\mathscr{M}(z), \Omega_{z}\right)\right\}_{0<|z|<r}$ be a family of nonsingular threefolds with nowhere vanishing holomorphic three-form, parametrized holomorphically by a punctured disk about the origin in the complex plane. Suppose that

(i) $\left(1-\mu^{k}\right)^{2}=0$ for some positive integer $k$.

(ii) For any locally constant homology class, $C(z) \in H_{3}(\mathscr{M}(z)), \int_{C(z)} \Omega_{z}$ is bounded along any ray, $\arg (z)=$ constant .

(iii) For any locally constant class $C(z) \in \operatorname{im}(1-\mu)$, and any fixed value of $\arg (z)$,

$$
\lim _{|z| \rightarrow 0}|z|^{-\epsilon} \int_{C(z)} \Omega_{z}=0
$$

for some $\epsilon>0$.

(iv) $\lim _{z \rightarrow 0} \int_{\mathscr{M}(z)} \Omega \wedge \bar{\Omega} \neq 0$ (it will follow from (i)-(iii) that the limit exists).

Then a smooth path in the disk has finite length in the Weil-Petersson metric, even if it passes through the origin.

Theorem B. Let $\mathscr{W}$ be a fourfold with nowhere vanishing holomorphic four-form $\Omega_{\mathscr{W}}$. Let $D$ be a disk around the origin and let $f: \mathscr{W} \rightarrow D$ be a proper holomorphic complex valued function on $\mathscr{W}$ whose fibres are compact and, except for $f^{-1}(0)$, nonsingular. Suppose further that the holomorphic three-form $\Omega_{z}$, defined along $\mathscr{M}(z)=f^{-1}(z)$ at all regular points by the relation

$$
\Omega_{z} \wedge d f=\Omega_{\mathscr{W}},
$$

is nowhere vanishing. Finally, suppose that the singularities of $\mathscr{M}(0)$ are all isolated and that each of them has a neighborhood in $\mathscr{W}$ which admits a coordinate system in which the coordinates may be weighted so that $f$ is weighted homogeneous with weight strictly lower than the sum of the weights of the coordinates. Then the holomorphic family $\left\{\left(\mathscr{M}(z), \Omega_{z}\right)\right\}_{z \in D-\{0\}}$ satisfies the hypotheses of Theorem A.

Proof of Theorem A. By passing to a $k$-fold cyclic cover of the punctured disk we may, without loss of generality, confine our discussion to the case $(1-\mu)^{2}=$ 0 . We observe next that we can regard $\Omega_{z}$ as an element of $H^{3}(\mathscr{M}(z), C)$, on which $\mu$ also operates by duality.

We write $\alpha=1-\mu$ and

$$
\hat{\Omega}_{z}=\Omega_{z}+\frac{\log (z)}{2 \pi i} \alpha\left(\Omega_{z}\right) .
$$

Although multiple valued, $\hat{\Omega}_{z}$ depends holomorphically on $z$ and satisfies the identity

$$
\Omega_{z}=\hat{\Omega}_{z}-\frac{1}{2 \pi i} \log (z) \alpha\left(\hat{\Omega}_{z}\right) .
$$

It follows by a straightforward computation that the periods of $\hat{\Omega}_{z}$ are singlevalued and therefore, by (ii), holomorphic in the unpunctured disk. Also by 
(iii), the periods of $\alpha\left(\hat{\Omega}_{z}\right)$ vanish at the origin. Now, for $z \neq 0$, we choose a locally constant symplectic basis $\left\{A_{i}(z), B_{i}(z)\right\}$ for $H_{3}(\mathscr{M}(z))$, and write

$$
\begin{gathered}
a_{i}(z)=\int_{A_{i}(z)} \hat{\Omega}_{z}, \quad b_{i}(z)=\int_{B_{i}(z)} \hat{\Omega}_{z}, \\
c_{i}(z)=\int_{A_{i}(z)} \alpha\left(\hat{\Omega}_{z}\right)=\int_{\alpha\left(A_{i}(z)\right)} \hat{\Omega}_{z}, \quad \text { and } \quad d_{i}(z)=\int_{B_{i}(z)} \alpha\left(\hat{\Omega}_{z}\right)=\int_{\alpha\left(B_{i}(z)\right)} \hat{\Omega}_{z} .
\end{gathered}
$$

We note that all these functions are single-valued and can be extended to be holomorphic in the unpunctured disk with $c_{i}(0)=d_{i}(0)=0$.

Next we avail ourselves of the identity,

$$
\int_{\mathscr{M}(z)} \Omega_{z} \wedge \bar{\Omega}_{z}=2 \mathfrak{J}\left(\sum_{i}\left(\int_{A_{i}(z)} \Omega_{z}\right)\left(\int_{B_{i}(z)} \bar{\Omega}_{z}\right)\right),
$$

of which the right-hand side can be rewritten,

$$
\text { (1) } 2 \mathfrak{J}\left(\sum_{i} a_{i} \bar{b}_{i}+\frac{\log (z)}{2 \pi i} \sum_{i} c_{i} \bar{b}_{i}-\frac{\log (\bar{z})}{2 \pi i} \sum_{i} a_{i} \bar{d}_{i}+\frac{\log (z) \log (\bar{z})}{4 \pi^{2}} \sum_{i} c_{i} \bar{d}_{i}\right) \text {. }
$$

Because (1) is single-valued, we may infer that the coefficients of $\arg (z)$ and $\arg (z)^{2}$ vanish identically. From the vanishing of the coefficient of $\arg (z)^{2}$ it follows that the last sum makes no contribution. The coefficient of $\arg (z)$ in (1) is

$$
\frac{1}{\pi} \mathfrak{J}\left(\sum_{i} c_{i} \bar{b}_{i}+\sum_{i} a_{i} \bar{d}_{i}\right)
$$

Hence

$$
\sum_{i} c_{i} \bar{b}_{i}+\sum_{i} a_{i} \bar{d}_{i}
$$

is identically real. Considering (2) as a power series in $z$ and $\bar{z}$ and recalling that $c_{i}(0)=d_{i}(0)=0$, we observe that there is no constant term, and that all terms of degree 0 in $z$ occur only in the right-hand sum while all terms of degree 0 in $\bar{z}$ occur only in the left-hand sum. Necessarily these terms are complex conjugates of one another and hence make no contribution to the coefficient of $\log |z|$ in (1), which is

$$
\frac{-1}{\pi} \mathfrak{R}\left(\sum_{i} c_{i} \bar{b}_{i}-\sum_{i} a_{i} \bar{d}_{i}\right) .
$$

It follows that (3) admits a factor of $z \bar{z}$, so that

$$
\int_{\mathscr{M}(z)} \Omega_{z} \wedge \bar{\Omega}_{z}=f(z, \bar{z})+z \bar{z} \log |z| g(z, \bar{z}),
$$

where $f$ and $g$ are real analytic in the disk, and $f(0) \neq 0$ by (iv). The finiteness of the Weil-Petersson distance to the origin follows by a standard computation. 
Proof of Theorem B. Let $\left\{\nu_{i}\right\}_{1 \leq i \leq n} \subset \mathscr{M}_{0}$ be the set of singular points of $f$. For each $\nu_{i}$ we choose coordinates $\left(x_{i}, y_{i}, u_{i}, v_{i}\right)$ with respective weights $\left(a_{i}, b_{i}, c_{i}, d_{i}\right)$, with respect to which $\nu_{i}$ is at the origin and $f$ is weighted homogeneous with weight $e_{i}<a_{i}+b_{i}+c_{i}+d_{i} . \Omega_{W}$ is given near $\nu_{i}$ by

$$
g_{i}(w) d x_{i} \wedge d y_{i} \wedge d u_{i} \wedge d v_{i}
$$

where $\left|g_{i}(w)\right|$ is bounded both above and below. For $w \in \mathscr{W}$ near $\nu_{i}$, we write

$$
\rho_{i}(w)=\left(\left(x_{i} \bar{x}_{i}\right)^{b_{i} c_{i} d_{i}}+\left(y_{i} \bar{y}_{i}\right)^{a_{i} c_{i} d_{i}}+\left(u_{i} \bar{u}_{i}\right)^{a_{i} b_{i} d_{i}}+\left(v_{i} \bar{v}_{i}\right)^{a_{i} b_{i} c_{i}}\right)^{1 / 2 a_{i} b_{i} c_{i} d_{i}} .
$$

For $r$ sufficiently small, we may define $B_{i}^{r}$ to be the locus of the inequality $\rho_{i}(w) \leq r . \quad B_{i}^{r}$ is diffeomorphic to a ball, and its boundary, which we shall denote $S_{i}^{r}$, is the locus of the equation $\rho_{i}(w)=r$ and is diffeomorphic to a sphere. We note that for $|\lambda| \leq 1$, the map

$$
h_{i}^{\lambda}:\left(x_{i}, y_{i}, u_{i}, v_{i}\right) \rightarrow\left(\lambda^{a_{i}} x_{i}, \lambda^{b_{i}} y_{i}, \lambda^{c_{i}} u_{i}, \lambda^{d_{i}} v_{i}\right)
$$

maps $\mathscr{M}_{z} \cap B_{i}^{r}$ holomorphically onto $\mathscr{M}_{\lambda^{e_{i} z}} \cap B_{i}^{|\lambda| r}$ and satisfies

$$
\left(h_{i}^{\lambda}\right)^{*}\left(\Omega_{\lambda_{i}^{e} z}\right)=\lambda^{a_{i}+b_{i}+c_{i}+d_{i}-e_{i}} \frac{g_{i} \circ h_{i}^{\lambda}}{g_{i}} \Omega_{z} .
$$

We fix "radii" $\left\{\rho_{i}^{0}\right\}$ so that the corresponding $B_{i}^{\rho_{i}^{0}}=B_{i}$ are defined in the appropriate coordinate neighborhoods and are disjoint. There is no loss of generality in assuming that $f\left(B_{i}\right)=D$ for each $i$, since we can simply restrict the remainder of the discussion to a smaller disk.

We write $\mathscr{W}_{i}$ for $\mathscr{W} \cap B_{i}$, and $\mathscr{W}_{\text {comp }}$ for the closure of $\mathscr{W}-\bigcup_{i} B_{i}$.

$$
\partial \mathscr{W}_{\text {comp }}=\bigcup_{i} \partial \mathscr{W}_{i}=\mathscr{W} \cap \bigcup_{i} S_{i},
$$

where $S_{i}$ is the boundary of $B_{i}$. We write $\mathscr{M}_{i}(z)$ and $\mathscr{M}_{\text {comp }}(z)$ respectively for $\mathscr{M}(z) \cap \mathscr{W}_{i}$ and $\mathscr{M}(z) \cap \mathscr{W}_{\text {comp }}$.

We observe that $\left(\mathscr{W}, \bigcup_{i} \mathscr{W}_{i}, \mathscr{W}_{\text {comp }}\right)$, with $\mathscr{M}(0)$ deleted, is a fiberwise triad over $D-\{0\}$ and that the pair $\left(\mathscr{W}_{\text {comp }}, \partial \mathscr{W}_{\text {comp }}\right)$ is a relative fibration over the full disk $D$. It follows that the Mayer-Vietoris sequences of the triads $\left(\mathscr{M}(z), \bigcup_{i} \mathscr{M}_{i}(z), \mathscr{M}_{\text {comp }}(z)\right)$ for distinct values of $z$ are identified locally, while the homology sequences of the pairs $\left(\mathscr{M}_{\text {comp }}(z), \partial \mathscr{M}_{\text {comp }}(z)\right)$ are identified globally. Moreover, the monodromy induces an automorphism of the MayerVietoris sequence of the triad and acts trivially on the homology sequence of the pair.

By combining the homology sequence of the pair, $\left(\mathscr{M}(z), \bigcup_{i} \mathscr{M}_{i}(z)\right)$ with the excision isomorphism

$$
H_{3}\left(\mathscr{M}_{\text {comp }}(z), \partial \mathscr{M}_{\text {comp }}(z)\right) \rightarrow H_{3}\left(\mathscr{M}(z), \bigcup_{i} \mathscr{M}_{i}(z)\right),
$$


we can see that

$$
(1-\mu)\left(H_{3}(\mathscr{M}(z))\right) \subseteq l_{*}\left(H_{3}\left(\bigcup_{i} \mathscr{M}_{i}(z)\right)\right),
$$

where $l$ is the inclusion of $\bigcup_{i} \mathscr{M}_{i}(z)$ in $\mathscr{M}(z)$.

If $C(z)$ is a locally constant homology class such that $C(z) \in l_{*} H_{3}\left(\mathscr{M}_{i}\left(z_{0}\right)\right)$, then we may choose $z_{0} \neq 0$ and represent $C\left(z_{0}\right)$ by an explicit piecewise smooth singular cycle $\mathscr{C}\left(z_{0}\right)$ on $\mathscr{M}_{i}\left(z_{0}\right)$. Then for $|\lambda| \leq 1, h_{i}^{\lambda} \circ \mathscr{C}\left(z_{0}\right)$ represents $C\left(\lambda^{e_{i}} z_{0}\right)$. One immediate consequence of this observation is

$$
C \in l_{*}\left(H_{3}\left(\mathscr{M}_{i}\right)\right) \Rightarrow \mu^{e_{i}}(C)=C .
$$

(6) and (7) together verify hypothesis (i) of Theorem A for $k=\operatorname{lcm}\left(\left\{e_{i}\right\}\right)$. We note next that for $0<r \leq 1$,

$$
\int_{h_{i}^{r} \circ \mathscr{C}\left(z_{0}\right)} \Omega_{r^{e} z_{0}}=\int_{\mathscr{C}\left(z_{0}\right)}\left(h_{i}^{r}\right)^{*}\left(\Omega_{r^{e} z_{0}}\right)=r^{a_{i}+b_{i}+c_{i}+d_{i}-e_{i}} \int_{\mathscr{C}\left(z_{0}\right)} \frac{g_{i} \circ h_{i}^{r}}{g_{i}} \Omega_{z_{0}},
$$

by (5). We write $\left|\Omega_{z_{0}}\right|$ for the real three-form on $\mathscr{C}\left(z_{0}\right)$ which differs from $\Omega_{z_{0}}$ by a unimodular factor and is compatible with the appropriate orientation on $\mathscr{C}\left(z_{0}\right)$. We also denote by $K_{i}$ a bound on $\left|g_{i}(w) / g_{i}\left(w^{\prime}\right)\right|$ for any $w, w^{\prime} \in B_{i}$. Such a bound exists since $\left|g_{i}(w)\right|$ is bounded both above and below. With this notation in place, we have

$$
\left|r^{a_{i}+b_{i}+c_{i}+d_{i}-e_{i}} \int_{\mathscr{C}\left(z_{0}\right)} \frac{g_{i} \circ h_{i}^{r}}{g_{i}} \Omega_{z_{0}}\right| \leq K_{i} r^{a_{i}+b_{i}+c_{i}+d_{i}-e_{i}} \int_{\mathscr{C}\left(z_{0}\right)}\left|\Omega_{z_{0}}\right|,
$$

which, together with (8), establishes hypothesis iii of Theorem A for

$$
\varepsilon<\min \left(\left\{\frac{a_{i}+b_{i}+c_{i}+d_{i}-e_{i}}{e_{i}}\right\}\right) .
$$

To address hypotheses (ii) of Theorem A, we let $C(z) \in H_{3}(\mathscr{M}(z)$ be locally constant and observe that we can represent $C\left(z_{0}\right)$ as a sum of singular chains

$$
\mathscr{C}_{\text {comp }}\left(z_{0}\right)+\sum_{i} \mathscr{C}_{i}\left(z_{0}\right)
$$

where $\mathscr{C}_{\text {comp }}\left(z_{0}\right)$ is a chain on $\mathscr{M}_{\text {comp }}\left(z_{0}\right), \mathscr{C}_{i}\left(z_{0}\right)$ is a chain on $\mathscr{M}_{i}\left(z_{0}\right)$ for each $i$, and

$$
\partial \mathscr{C}_{\text {comp }}\left(z_{0}\right)=-\sum_{i} \partial \mathscr{C}_{i}\left(z_{0}\right)
$$

Next, we extend $\mathscr{C}_{\text {comp }}\left(z_{0}\right)$ continuously to a chain $\mathscr{C}_{\text {comp }}\left(r z_{0}\right)$ on $\mathscr{M}_{\text {comp }}\left(r z_{0}\right)$ such that $\partial \mathscr{C}_{\text {comp }}\left(r z_{0}\right)$ is a cycle on $\partial \mathscr{M}_{\text {comp }}\left(r z_{0}\right)$ for $0 \leq r \leq 1$, using the triviality of the relative fibration $\left(\mathscr{W}_{\text {comp }}, \partial \mathscr{W}_{\text {comp }}\right)$ over the disk $D$. We have

$$
\int_{C\left(z_{0}\right)} \Omega_{z_{0}}=\int_{\mathscr{C}_{\text {comp }}\left(z_{0}\right)} \Omega_{z_{0}}+\sum_{i} \int_{\mathscr{C}_{i}\left(z_{0}\right)} O m_{z_{0}}
$$


Also

$$
\int_{\mathscr{C}_{\text {comp }}\left(r z_{0}\right)} \Omega_{r z_{0}}
$$

is continous for $0 \leq r \leq 1$, and therefore bounded. Now we set

$$
\mathscr{E}_{i}=\mathscr{C}_{i}\left(z_{0}\right) \cup \bigcup_{0 \leq r \leq 1}\left(\partial \mathscr{C}_{\text {comp }}\left(r z_{0}\right) \cap B_{i}\right),
$$

and

$$
\mathscr{K}_{i}=\left\{\nu_{i}\right\} \cup \bigcup_{0<r \leq 1} h_{i}^{r}\left(\mathscr{E}_{i}\right) .
$$

For each $i$ and for $0 \leq r \leq 1$ choose $\mathscr{C}_{i}\left(r z_{0}\right)$ to be a triangulation of $\mathscr{M}_{i} \cap \mathscr{K}_{i}$, oriented so that

$$
\partial \mathscr{C}_{\text {comp }}\left(r z_{0}\right)=-\sum_{i} \partial \mathscr{C}_{i}\left(r z_{0}\right)
$$

and hence

$$
\int_{C\left(r z_{0}\right)} \Omega_{r z_{0}}=\int_{\mathscr{E}_{\text {comp }}\left(r z_{0}\right)} \Omega_{r z_{0}}+\sum_{i} \int_{\mathscr{E}_{i}\left(r z_{0}\right)} \Omega_{r z_{0}}
$$

Moreover

$$
h_{i}^{r}\left(\left|\mathscr{C}_{i}\left(z_{0}\right)\right|\right)=\left|\mathscr{C}_{i}\left(r^{e_{i}} z_{0}\right)\right| \cap h_{i}^{r}\left(B_{i}\right) .
$$

where $|\mathscr{C}|$ denotes the support of a chain. By (14), we have

$$
\int_{\mathscr{C}_{i}\left(r^{e_{i} z}\right)}\left|\Omega_{r^{e_{i} z}}\right|=\int_{\mathscr{E}_{i}\left(r^{e_{i} z}\right) \cap h_{i}^{r}\left(B_{i}\right)}\left|\Omega_{r^{e_{i}} z_{0}}\right|+\int_{\mathscr{C}_{i}\left(r^{e_{i} z}\right)-h_{i}^{r}\left(B_{i}\right)}\left|\Omega_{r^{e_{i} z}}\right|,
$$

where $r>0,0<z / z_{0} \leq 1$, and $|\Omega|$ is defined as preceding (9). The domain of the right hand integral is bounded away from $\nu_{i}$ even for $z=0$. It follows that this integral is proper and continuous at $z=0$ and so is bounded, say by I . Analogous to (9), we also have

$$
\int_{\mathscr{C}_{i}\left(r^{\left.e_{i} z\right) \cap h_{i}^{r}\left(B_{i}\right)}\right.}\left|\Omega_{r^{e_{i}}}\right| \leq K_{i} r^{a_{i}+b_{i}+c_{i}+d_{i}-e_{i}} \int_{\mathscr{C}_{i}(z)}\left|\Omega_{z}\right| .
$$

We fix $\mathbf{r}$ so that $0<\mathbf{r}<1$ and $\mathbf{R}=K_{i} \mathbf{r}^{a_{i}+b_{i}+c_{i}+d_{i}-e_{i}}<1$. It now follows from (15) and (16) that

$$
\int_{\mathscr{E}_{i}\left(\mathbf{r}^{\left.e_{i} z\right)}\right.}\left|\Omega_{\mathbf{r}^{e_{i}}}\right| \leq I+\mathbf{R} \int_{\mathscr{E}_{i}(z)}\left|\Omega_{z}\right|
$$

and hence, by induction, that

$$
\int_{\mathscr{C}_{i}\left(\mathbf{r}^{\left.k e_{i} z\right)}\right.}\left|\Omega_{\mathbf{r}^{k e_{i}}}\right| \leq I \sum_{j=0}^{k-i} \mathbf{R}^{j}+\mathbf{R}^{k} \int_{\mathscr{C}_{i}(z)}\left|\Omega_{z}\right| .
$$

Finally, we let $J$ be a bound on $\int_{\mathscr{C}_{i}\left(r z_{0}\right)}\left|\Omega_{r z_{0}}\right|$ for $\mathbf{r} \leq r \leq 1$. Then it follows from (18) that

$$
\int_{\mathscr{C}_{i}\left(r z_{0}\right)}\left|\Omega_{r z_{0}}\right| \leq \frac{1}{1-\mathbf{R}} \max \{I, J\}, \quad 0<r \leq 1 .
$$


Hypothesis (ii) of Theorem A now follows from (13) and (19). The verification of hypothesis (iv) of Theorem A follows from the continuity throughout $D$ of

$$
\int_{\mathscr{M}_{\text {comp }}(z)} \Omega_{z} \wedge \bar{\Omega}_{z},
$$

which does not vanish at $z=0$. This completes the proof of Theorem B.

Theorem B applies most directly to degenerations of Calabi-Yau threefolds realized as hypersurfaces in an algebraic fourfold. Let $\mathscr{Z}$ be a fourfold, $\xi$ be a section of $K_{\mathscr{L}}^{*}$ with isolated singularities satisfying the homogeneity hypothesis of Theorem B, and $\eta$ be a nonsingular section of $K_{\mathscr{L}}^{*}$ which is nonvanishing at the singular points of $\xi$. Let $\Omega_{\mathscr{L}}$ be a local section of $K_{\mathscr{L}}, h=\left\langle\xi, \Omega_{\mathscr{L}}\right\rangle$ and $g=\left\langle\eta, \Omega_{\mathscr{X}}\right\rangle$. It is straightforward to verify that if we restrict $z$ to be sufficiently small and set $\mathscr{W}=\{(x, z) \mid(\xi+z \eta)(x)=0\}$ with $f(x, z)=z$ and $\Omega_{\mathscr{W}}$ defined by

$$
\Omega_{\mathscr{W}} \wedge(d h+z d g)=\Omega_{\mathscr{Z}} \wedge d z
$$

then $\Omega_{\mathscr{W}}$ is independent of the choice of $\Omega_{\mathscr{Z}}$ and therefore global, and $\mathscr{W}$, $f$ and $\Omega_{\mathscr{W}}$ satisfy the hypotheses of Theorem B.

\section{REFERENCES}

1. P. Candelas, P. S. Green, and T. Hübsch, Connected Calabi-Yau compactifications, Strings 1988, World Scientific (to appear).

2. K. Kodaira, Complex manifolds and deformations of complex structures, Springer, Berlin, Heidelberg, and New York, 1986.

Department of Mathematics, University of Maryland, College Park, Maryland 20742 